Check for updates

1 University of Oxford.

2 University of California, San Francisco.

3 Oxford and London

Twitter: @HannahChase41 @HampshireKarly

Cite this as: BMJ 2022;376:0209

http://dx.doi.org/10.1136/bmj.0209

Published: 25 January 2022

\section{Improving the medical curriculum on planetary health and sustainable healthcare}

\section{We urgently need to educate our doctors and students on the accelerating climate emergency and on how to create a sustainable healthcare system}

\author{
Hannah Chase, ${ }^{1}$ Karly Hampshire, ${ }^{2}$ SanYuMay Tun ${ }^{3}$
}

The planetary health framework links human health to the health of the planet, which is our life support system. The most recent Intergovernmental Panel on Climate Change (IPCC) Report (August 2021) emphasises, once again, that the climate crisis is the greatest threat to health this century. ${ }^{1}$ Paradoxically, healthcare delivery has an adverse impact on the environment, and therefore health. If the healthcare system were a country, it would be the fifth largest carbon emitter. ${ }^{2}$ Therefore, health professionals have to learn sustainable healthcare in order to protect planetary health. Education for Sustainable Healthcare (ESH) is defined as the process of equipping current and future health professionals with the knowledge, values, confidence, and capacity to provide sustainable healthcare services, including mainstreaming planetary health as an integrated curricular theme. ${ }^{3}$

Why should ESH be part of every doctor's education? Patients reach out to doctors to discuss disease aetiology and risk factors, and yet, most doctors are not knowledgeable on the greatest health risk their patients currently face, even as they treat increasing numbers of patients already affected by the health impacts of climate change. As a highly trusted profession with an important leadership role within wider society, doctors must communicate accurate information to patients and should be empowered to influence societal changes at policy level.

Furthermore, if the NHS is going to achieve its net-zero commitments, such as reaching net zero by 2040 for NHS England, the workforce plays a crucial role in driving reform.

At all levels of the medical education system, ESH training is inadequate and needs to be improved. In the UK, the General Medical Council (GMC) Outcomes for graduates guidance recently added stipulations to include teaching on sustainable healthcare. 45 However, the GMC does not, as yet, specifically require education on planetary health. At the postgraduate level, there is an "Environmentally Sustainable Healthcare" module on the national online training platform, but this is optional. Many educational bodies are recognising the importance of ESH, for example by hosting a public lecture series, or declaring a climate emergency. ${ }^{67}$ However, in the specialty training pathways there is still no in-depth compulsory education on sustainable healthcare although some are actively working towards this goal. Recent global data indicate that only $15 \%$ of medical schools deliver teaching on climate change, but the study did not go into detail about what this entailed or cover sustainable healthcare teaching. ${ }^{8}$ We developed the Planetary Health Report Card to help catalyse an educational shift and to fill this gap. It is a student-led project for evaluating medical school engagement in planetary health and sustainability. ${ }^{9}$ The PHRC is a metric-based initiative which assesses medical schools in five areas: curriculum, research, community outreach and advocacy, support for student-led initiatives, and campus sustainability. The second annual report was published in April 2021, evaluating 62 medical schools in five nations (U.S, Canada, UK, Republic of Ireland, and Malaysia). The results confirmed inadequate education and engagement in planetary health and sustainable healthcare by medical schools. The curriculum section was notably weak in all nations and, even in the UK where there is the regulatory requirement to teach on sustainable healthcare, a third of the 30 participating UK medical schools had no teaching at all on sustainable healthcare in their core curriculum.

Medical schools have paid considerable attention to the PHRC since its inception. Combined with the energy surrounding $\mathrm{COP} 26$, the NHS Net Zero targets and GMC requirements, the PHRC has sparked a timely change in medical school agendas. Many schools internationally have already made permanent changes to their core curriculum and formed PHRC steering groups to integrate teaching throughout the curriculum.

The PHRC facilitates the sharing of ideas and inspires change through open access, direct comparison of different schools on the website and descriptive explanations of individual results, including detailed examples of good practice. We suggest that a similar approach could be effective to catalyse the urgent changes required for postgraduate and other health professional training pathways. Already, we are supporting the development of international PHRCs adapted for nursing and pharmacy training programmes.

The world is rapidly changing, and the current generation of health professionals need to understand the impacts of the climate emergency on health and develop the skills of adaptation and mitigation to promote planetary health. In the healthcare sphere, mitigation includes designing and working in a zero-carbon healthcare system. To achieve this Education for Sustainable Healthcare is a fundamental need in all healthcare professions globally and at all levels of training. This education is currently insufficient and the IPCC report only adds to this urgency. 


\section{OPINION}

Competing interests: HC is co-director of the Planetary Health Report Card and was UK-lead in 2020. $\mathrm{KH}$ is the founding co-director of the Planetary Health Report Card initiative and a current fellow with the new University of California Center for Climate, Health, and Equity. ST is UK advisor to the PHRC. She teaches Planetary Health and Sustainable Healthcare at undergraduate, postgraduate and faculty development levels and is involved internationally in embedding this into health professions education.

Provenance and peer review: Not commissioned, not peer reviewed

1 The Intergovernmental Panel on Climate Change. AR6 Climate Change 2021: The Physical Science Basis. 2021. https://www.ipcc.ch/report/sixth-assessment-report-working-group-i/

2 Health Care Without Harm. Healthcare climate footprint report. 2019.

3 Shaw E, Walpole S, McLean M, etal. AMEE Consensus Statement: Planetary health and education for sustainable healthcare. Med Teach 2021;43:272-86.

doi: 10.1080/0142159X.2020.1860207. pmid: 33602043

4 General Medical Council. Outcomes for Graduates 2018. London: 2018

5 Tun S. Fulfilling a new obligation: Teaching and learning of sustainable healthcare in the medical education curriculum. Med Teach 2019:41:1168-77.

doi: 10.1080/0142159X.2019.1623870. pmid: 31237167

6 The Royal Society of Medicine. The Health Emergency of Climate Change Series. 2021. https://www.rsm.ac.uk/latest-news/2021/climate-change-series-resources-on-health-and-climatechange/ (accessed 27 Oct 2021).

7 Health Declares. https://healthdeclares.org/declarations/ (accessed 27 Oct 2021)

8 Omrani OE, Dafallah A, Paniello Castillo B, etal. Envisioning planetary health in every medical curriculum: An international medical student organization's perspective. Med Teach 2020;42:1107-11. doi: 10.1080/0142159X.2020.1796949. pmid: 32757869

9 Hampshire K, Chase H, Islam N, et al. Planetary Health Report Card. www.phreportcard.org (accessed 4 Nov 2021) 